\title{
Exploring Bebras Tasks Content and Performance: A Multinational Study
}

\author{
Cruz IZU1 ${ }^{1}$, Claudio MIROLO ${ }^{2}$, Amber SETTLE ${ }^{3}$ \\ Linda MANNILA ${ }^{4}$, Gabriele STUPURIENE ${ }^{5}$ \\ ${ }^{1}$ The University of Adelaide, Adelaide, Australia \\ ${ }^{2}$ University of Udine, Udine, Italy \\ ${ }^{3}$ DePaul University, Chicago, USA \\ ${ }^{4}$ Linköping University, Linköping, Sweden \\ ${ }^{5}$ Institute of Mathematics and Informatics, Vilnius University, Vilnius, Lithuania \\ e-mail:cruz@cs.adelaide.edu.au,claudio.mirolo@uniud.it,asettle@cdm.depaul.edu, \\ linda.mannila@liu.se,gabriele.stupuriene@mii.vu.lt
}

Received: October 2016

\begin{abstract}
Although there is no universal agreement that students should learn programming, many countries have reached a consensus on the need to expose K-12 students to Computational Thinking (CT). When, what and how to teach CT in schools are open questions and we attempt to address them by examining how well students around the world solved problems in recent Bebras challenges. We collected and analyzed performance data on Bebras tasks from 115,400 students in grades 3-12 in seven countries. Our study provides further insight into a range of questions addressed in smaller-scale inquiries, in particular about the possible impact of schools systems and gender on students' success rate.

In addition to analyzing performance data of a large population, we have classified the considered tasks in terms of CT categories, which should account for the learning implications of the challenge. Algorithms and data representation dominate the challenge, accounting for $75-90 \%$ of the tasks, while other categories such as abstraction, parallelization and problem decomposition are sometimes represented by one or two questions at various age groups. This classification can be a starting point for using online Bebras tasks to support the effective learning of CT concepts in the classroom.
\end{abstract}

Keywords: computational thinking, Bebras challenge, task difficulty, gender issues.

\section{Introduction}

Regardless of the variety of approaches, there seems to be "a convergence towards computational thinking as a core idea of the K-12 curricula" (Hubwieser et al., 2014). The term computational thinking (CT) was coined by Papert (Papert, 1980) but gained increased attention beginning in the 2000s after Wing published her seminal article posi- 
tioning $\mathrm{CT}$ as a "universally applicable attitude and skill set everyone, not just computer scientists, would be eager to learn and use" (Wing, 2006). Since then, many countries have introduced in their school curricula CT or other computer science topics, including programming (McCartney and Tenenberg, Eds., 2014, Barendsen and Steenvoorden, 2016, Sentance et al., 2013, Tort and Drot-Delange, 2013, Barendsen et al., 2016, Heintz et al., 2016).

CT is meant to encompass a set of concepts and thought processes that aid in approaching problems arising in different fields in a way that could involve computers (Mannila et al., 2014). However, the concepts central to $\mathrm{CT}$ can be quite foreign to many K-12 teachers. Thus, among other challenges, it is important to get both teachers and students engaged by developing learning materials that can provide a positive first experience. A compelling example of this kind of material is provided by the Bebras community, which annually arranges an international problem-solving challenge aimed at children and youth aged 8-18 (Dagienè and Sentance, 2016, Bezáková and Winczer, 2011). The challenge includes motivating and age-adapted tasks relating to, for instance, programming and algorithms, computational thinking and information technology. Moreover, it does not require any background in these areas. All material from past challenges are available online freely for anyone to use, and each task includes specific information not only on the correct answer, but also on the connection to computer science topics.

A working group at the ITiCSE 2015 conference started an exploratory investigation of the "task based assessment" approach of the Bebras challenge within the broader framework of K-9 computer science education, which encompassed the development of national curricula and guidelines as well as teachers' attitudes toward their practice in this field (Barendsen et al., 2015). In particular, their final report proposed a classification of the conceptual content and question structure of Bebras tasks from years 2010-2014 according to CT categories. Algorithms and data representation were found to be the main concept categories in the K-9 context. Moreover, a taxonomy for questions related to algorithms was outlined.

In this study, we have analyzed in terms of concept coverage, presumed ${ }^{1}$ and actual performance a selection of Bebras tasks for seven countries based on data collected in 2014 and 2015. Given that many countries are in the process of updating their curricula, it is likely that the Bebras challenge was one of the first opportunities for students in those countries to be exposed to CT.

The study extends previous work on Bebras tasks (see subsection 2.2) by answering the following research questions based on large multinational cohorts:

- Which CT concepts and skills are addressed by Bebras tasks?

- Is estimated task difficulty related to the CT conceptual content? how well does the estimated difficulty match the observed students' performance?

- Is gender a significant factor in performance for any country or age group?

The first question is important in order to explore the implications of CT conceptual content both in terms of curricular coverage and cognitive demand. The aim of the sec-

\footnotetext{
${ }^{1}$ According to the difficulty level assigned by the task proponents.
} 
ond question is to identify whether some concepts and tasks appear to be more difficult for student and hence might need more scaffolding when introduced at school. Finally, the third question is important in order to contribute to the knowledge and discussion on raising girls' interest in computer science. All in all, addressing these research questions will reveal to what extent Bebras tasks match the need for suitable CT teaching material in $\mathrm{K}-12$ education.

By analyzing performance data of a large population and from rather different contexts, our study provides further insight into a range of questions recently addressed in smaller-scale inquiries, in particular about the possible impact of schools systems and gender on learning CT concepts. Distinctive characters of our present work are indeed the cohort size, amounting to 115,400 students in grades $3-12$, as well as the variety of educational contexts in the seven countries that have been considered. In addition, the paper represents one of the first attempts to explore the implications of the overall conceptual content of Bebras tasks within a CT-related framework.

The rest of the paper is organized as follows: we first introduce the Bebras challenge and review previous work studies relevant for the present analysis. Then we describe the set of data collected, and analyze them both qualitatively and quantitatively in terms the concept coverage, performance and difficulty level of a selection of the tasks. Finally, we end with some conclusions and recommendations.

\section{Background}

\subsection{The Bebras Challenge}

The Bebras challenge ${ }^{2}$ is an online competition that was introduced in 2004 in Lithuania as an outreach instrument, to encourage interest and motivation in computing-related topics (Dagienè, 2006). Since then, it has spread worldwide reaching 1.3 million of pupils from 38 countries in the autumn 2015's challenge and more than 1.5 million from 30 countries in the 2016's challenge.

Bebras tasks are short and answerable in a few minutes through a computerized interface. The task set is created on a yearly basis through crowd-sourcing from participating countries, which are then further reviewed and polished during an annual workshop (Dagienè and Stupurienè, 2016). Most of the tasks we have analyzed are either multichoice or interactive. Multi-choice tasks have four nontrivial and well-defined answer choices with only one correct solution. Interactive tasks provide a scene or diagram on the screen that participants can interact with to carry out actions and change the scene's state to move towards the question's goal; this interaction may provide feedback on the status of the task.

The challenge is arranged annually in their local languages throughout the world. Most countries run their event on Bebras Week, which falls on the second full week of November (southern hemisphere countries may use a different week).

\footnotetext{
${ }^{2}$ http://www. bebras.org
} 
Participants are divided into age groups: Mini (grades 3-4); Benjamin (grades 5-6); Cadet (grades 7-8); Junior (grades 9-10); Senior (grades 11-12). The grades corresponding to the age groups may differ slightly between countries based on their local curricula $^{3}$. Each age group attempts 15 (9 for Mini) to 18 tasks within 45-55 minutes. They will have the same number of tasks, usually 5 or 6 tasks, at each difficulty level: A (easy), B (medium) and C (hard) as prescribed by developers. The participants are usually supervised by teachers who may integrate the challenge in their teaching activities.

\subsection{Related Work}

The popularity of the Bebras' initiative provides the opportunity to evaluate its results in a variety of contexts; this also prompted discussions about the possible implications for K-12 computer science education. There is already quite an extensive review of work related to the Bebras challenge (Dagienè and Stupurienè, 2016). Therefore, in this subsection we will focus on the contributions addressing tasks' scope and difficulty. We also discuss previous work done on CT classification since such work is relevant to the qualitative analysis of the Bebras tasks discussed later in the paper.

Besides their outreach potential (Haberman et al., 2011, Bell et al., 2011), Bebras tasks can be incorporated in the curriculum (Dagienè and Sentance, 2016) or used as an instrument to support teachers' training in computing subjects (Bezáková and Winczer, 2011), as well as to engage students with CT in connection with their informal training for the contest. Gujberova and Kalas present an example of this perspective in order to introduce "modern Informatics as opposed to ICT classes" in K-12 education (Gujberova and Kalas, 2013). It is especially from this standpoint that the Bebras challenge is an interesting topic of study.

As discussed in (Kalelioglu et al., 2016), there are a wide range of papers in recent years that cover the definitions and classification of Computational Thinking. A key source of classification for CT problems is the Computer Science Teachers Association ${ }^{4}$ (CSTA), which has published in cooperation with the ISTE $^{5}$ a "Computational Thinking Across the Curriculum" table introducing their list of CT categories. In fact, we followed the CT characterization proposed in previous work (Barendsen et al., 2015), which is inspired by these CSTA/ISTE categories: data collection, data analysis, data representation, problem decomposition, abstraction, algorithms \& procedures, automation, parallelization and simulation. The detailed definitions and operational characterization that were used for the qualitative analysis are provided in Table 1.

Bebras tasks are becoming a popular tool to assess computer science competences at various levels of school instruction (Duncan and Bell, 2015), to the point that Hubwieser and Mühling are envisaging a possible future role in PISA-like assessment of such

\footnotetext{
${ }^{3}$ In particular, South Africa uses also a different labeling of age groups, which we mapped as follows in order to have a one-to-one correspondence: Junior (grade 7) $\rightarrow$ Benjamin, Intermediate (8-9) $\rightarrow$ Cadet, Senior (10-11) $\rightarrow$ Junior and Elite $(12) \rightarrow$ Senior. Other countries not considered in this study have opted for different arrangements and/or larger sets of tasks.

${ }^{4}$ http://www.csteachers.org

${ }^{5}$ https://www. iste.org
} 
Table 1

CT definitions from previous work (Barendsen et al., 2015)

\begin{tabular}{|c|c|c|}
\hline CT category & Definition & Operational definition \\
\hline Data collection & $\begin{array}{l}\text { The process of gathering app- } \\
\text { ropriate information. }\end{array}$ & Find a data source for a problem area. \\
\hline Data analysis & $\begin{array}{l}\text { Making sense of data, fin- } \\
\text { ding patterns, and drawing } \\
\text { conclusions. }\end{array}$ & $\begin{array}{l}\text { Take data and transform it to solve a problem. Often there } \\
\text { is some statistical analysis involved in the transformation, } \\
\text { although the statistics do not have to be sophisticated. }\end{array}$ \\
\hline $\begin{array}{l}\text { Data } \\
\text { representation }\end{array}$ & $\begin{array}{l}\text { Depicting and organizing data } \\
\text { in appropriate graphs, charts, } \\
\text { words, or images. }\end{array}$ & $\begin{array}{l}\text { Take data and put it into a specified format. Includes } \\
\text { descriptions of data that involve particular structures. It } \\
\text { may involve understanding the implications of graphs or } \\
\text { other representations on the solution of a problem. }\end{array}$ \\
\hline $\begin{array}{l}\text { Problem } \\
\text { decomposition }\end{array}$ & $\begin{array}{l}\text { Breaking down tasks into } \\
\text { smaller, manageable parts. }\end{array}$ & $\begin{array}{l}\text { Breaking a problem or task into smaller pieces to enable an } \\
\text { easier or better solution. }\end{array}$ \\
\hline Abstraction & $\begin{array}{l}\text { Reducing complexity to de- } \\
\text { fine main idea. }\end{array}$ & $\begin{array}{l}\text { Problems that ask for the creation of a formula. The } \\
\text { distillation of broader ideas out of narrower concepts. } \\
\text { Finding rules that apply to a given problem. Finding a } \\
\text { pattern to model some behavior. Identifying essential facts } \\
\text { about a structure or problem to verify correct answers. }\end{array}$ \\
\hline $\begin{array}{l}\text { Algorithms \& } \\
\text { procedures }\end{array}$ & $\begin{array}{l}\text { Series of ordered steps taken } \\
\text { to solve a problem or achieve } \\
\text { some end. }\end{array}$ & $\begin{array}{l}\text { Solving maximization, minimization, or other optimization } \\
\text { problems. Following a step-by-step procedure. Verifying } \\
\text { potential solutions as valid or invalid. Encoding or } \\
\text { encryption/decryption problems, including the application } \\
\text { of an encryption scheme to a sample set of data. Debugging } \\
\text { solutions and finding errors in a solution. Applying a set of } \\
\text { rules to determine specific values. Choosing or verifying } \\
\text { pseudocode or code. }\end{array}$ \\
\hline Automation & $\begin{array}{l}\text { Having computers or ma- } \\
\text { chines do repetitive or tedious } \\
\text { tasks. }\end{array}$ & No instances found. \\
\hline Parallelization & $\begin{array}{l}\text { Organize resources to simul- } \\
\text { taneously carry out tasks to } \\
\text { reach a common goal. }\end{array}$ & Scheduling problems. \\
\hline Simulation & $\begin{array}{l}\text { Representation of a model } \\
\text { of a process. Simulation also } \\
\text { involves running experiments } \\
\text { using models. }\end{array}$ & $\begin{array}{l}\text { Tasks that are interactive and involved building and } \\
\text { exploring a solution. }\end{array}$ \\
\hline
\end{tabular}

competences (Hubwieser and Mühling, 2014). However, multiple studies have identified the apparent discrepancy between a task's estimated and perceived difficulty as a major concern. Yagunova and colleagues found a correlation between length of statement and difficulty for primary school students, and proposed using an "a posteriori task difficulty to compensate for differences" (Yagunova et al., 2015). Other researchers investigated the psychometric factors which influence success (Hubwieser and Mühling, 2015), explored how to reliably predict task difficulty (van der Vegt, 2013), and developed statistical models for this purpose (Bellettini et al., 2015).

By exploring the performance of three European countries, Dagienè et al. (Dagienè et al., 2014) found that the ability to solve tasks was largely independent of the school system, and that often supposedly easy tasks turned out to be harder than expected to the lower age categories. 
Bebras performance has also been analyzed from a gender perspective, but the findings appear to be still inconclusive. On the one hand, Dagienè and colleagues were not able to uncover any significant difference in overall performance (Dagienè et al., 2014), although a study of the 2014 Lithuanian contest showed that boys outperformed girls in specific tasks requiring spatial reasoning and were more likely to guess an uncertain answer than girls (Dagienè et al., 2015). On the other hand, an investigation of a large cohort taking part in the 2014 German challenge revealed that boys of all age groups performed appreciably better compared to girls, the gap increasing strikingly for the eldest students (Hubwieser et al., 2016).

\section{Methodology}

\subsection{Data Collection}

Bebras contest data are stored on local servers and initially we had access to anonymized data from four countries (Italy, Australia, Finland, and Lithuania); we also received results (percentage of correct answers per age group and gender) from South Africa, Switzerland and Canada.

The list of countries and their participation numbers are listed in Table 2. Finland (FIN), Lithuania (LIT) and Canada (CAN) provided data for both years. Australia (AUS) and South Africa (SAf) ran their challenges in early 2015, but they are listed in 2014 as they used 2014 tasks. Switzerland (SW) and Italy (ITA) provided data for their

Table 2

Participation data for 2014 and 2015, covering 115,400 students

\begin{tabular}{lrrrrrr}
\hline $\mathbf{2 0 1 4}$ & AUS & FIN & LIT & SAf & CAN & Total \\
\hline Mini & 1837 & 585 & 2409 & - & - & 9,589 \\
Benjamin & 2350 & 675 & 7167 & 1558 & - & 10,571 \\
Cadet & 2568 & 1322 & 5988 & 3059 & - & 13,214 \\
Junior & 1344 & 2140 & 6265 & 2602 & 4558 & 13,791 \\
Senior & 526 & 128 & 3147 & 999 & - & 4,062 \\
\hline & 8625 & 4850 & 24976 & 8218 & 4558 & $\mathbf{5 1 , 2 2 7}$ \\
\hline $\mathbf{2 0 1 5}$ & ITA & FIN & LIT & SW & CAN & Total \\
\hline Mini & 1648 & 702 & 2374 & 1026 & - & 10,486 \\
Benjamin & 904 & 2527 & 7110 & 1947 & - & 11,188 \\
Cadet & 1622 & 1425 & 5810 & 4905 & 4638 & 18,704 \\
Junior & 2083 & 3735 & 6114 & 4124 & 5713 & 18,959 \\
Senior & 287 & 1207 & 3304 & 1411 & - & 5,279 \\
\hline & 6544 & 9596 & 24712 & 13413 & 9908 & $\mathbf{6 4 , 1 7 3}$ \\
\hline
\end{tabular}


2015 challenges $^{6}$. Thus, we have results from a total of 115,400 students from seven different countries.

There is a large Bebras task pool for each given year, out of which each country selects their own local subset (usually 15 questions, 18 for Lithuania) for each age group. Some countries use smaller sets, 9 to 12 questions, for the Mini age-group. The collection of Bebras tasks used in 2014-15 by the countries listed in Table 2 had 145 different tasks, of which 88 were used by 2 or more countries.

\subsection{Qualitative Analysis}

In order to address the research questions presented in section 1, we needed to look at the data from both a qualitative and a quantitative perspective. As mentioned above (subsection 2.2), we based our CT classification on a framework developed in the previous work (Barendsen et al., 2015), summarized in Table 1.

The qualitative study was done through content analysis, see e.g. (Cohen et al., 2013, Gavora, 2015), in which either two or three of the authors independently went through all selected tasks and assigned each task one or more of the CT categories summarized in Table 1. The authors agreed on the majority of assigned categories; in cases of disagreement, one of the authors gave a suggestion for how to resolve the situation, which could then be accepted or countered with another suggestion by the other authors.

After three revisions and related discussion about the most appropriate characterization of the tasks assigned in 2014 and 2015 (in the considered countries), an inter-rater agreement of $90.2 \%$ was attained. A final decision was made by one of the co-authors chosen by the others to review and arbitrate disagreements between the rest. None of the resulting classifications were disputed by the group as a whole. We may also notice that our classification is not always in agreement with the proponents' intentions, as attested by the section "it's informatics" in the task documentation?. More specifically, full agreement between both raters and proponents is attained for about $59 \%$ of the tasks, whereas there is a complete mismatch in $4 \%$ of the cases and some substantial difference in further $7 \%$.

Next, we provide specific examples of the classification to illustrate both the process and the way that the CT categories were instantiated in the Bebras tasks.

Exercise 2015-MY-01 ${ }^{8}$ shows a broken bracelet made out of six colored beads with different shapes as shown in Fig. 1. The student must pick the corresponding bracelet out of the four given circular ones.

This task was classified as data representation (DR). This is because the key to solving the task is to realize that the circular representation of each of the solutions imposes conditions regarding which shapes can be located next to each other. Once the importance of adjacency of shapes is recognized, it is straightforward to verify which option is the correct one.

\footnotetext{
${ }^{6}$ Italy ran the challenge in teams of 3-4, but the table reects the total number of students.

${ }^{7}$ Actually, the same task may be categorized differently, or with a different emphasis on its informatics/CT features, in the documentation provided by organizers from different countries.

${ }^{8}$ Each task is identified by three data: year, proponent country and progressive number.
} 


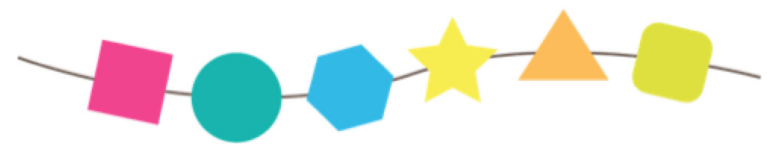

a)

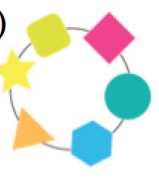

b)

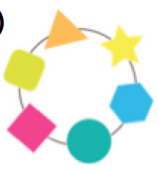

c)

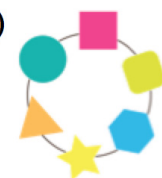

d)

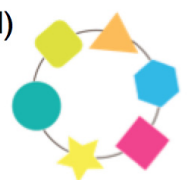

Fig. 1. Task 2015-MY-01.

Exercise 2015-LT-03 describes an automated system in a chocolate factory. The commands that can be given to the decorating robot arm are: Leaf - draws a long oval, Circle - draws a circle, Rotate $k$ - rotates the sweet clockwise by $\mathrm{k}$ degrees, and Repeat $n[\ldots]-$ repeats the commands in the brackets $n$ times.

An example is given of a sequence of commands and the drawing it would produce. Then the drawing in Fig. 2 is provided and the question asks which of the following command sequences does not cause the robot to draw the indicated flower. The options are:

A. Repeat 6 [Rotate 30 Circle Rotate 30 Leaf].

B. Repeat 6 [Leaf Rotate 60] Rotate 330 Repeat 6 [Circle Rotate 300].

C. Repeat 6 [Leaf Rotate 60] Repeat 6 [Circle Rotate 60].

D. Repeat 3 [Rotate 120 Repeat 2 [Leaf Rotate 30 Circle Rotate 150]].

This task was classified as algorithms \& procedures (ALG). The problem has provided various functions defined for the robot and asks which sequence of function calls will produce the correct drawing. While the use of procedures might suggest abstraction, the exercise does not call for the creation of any procedures. Instead, the problem is asking for interpretation and/or verification of a sequence of commands, something we classified as algorithms.

Exercise 2015-CZ-01 provides an example of a Bebras task that was classified into multiple CT categories. The exercise asks students to match the "walnut animals" in the two rows of Fig. 3 - i.e., to recognize graph isomorphism. This task was classified both as data representation (DR) and as abstraction (ABS), the latter because in order to match isomorphic graphs students are required to think of their structure at a more abstract level.
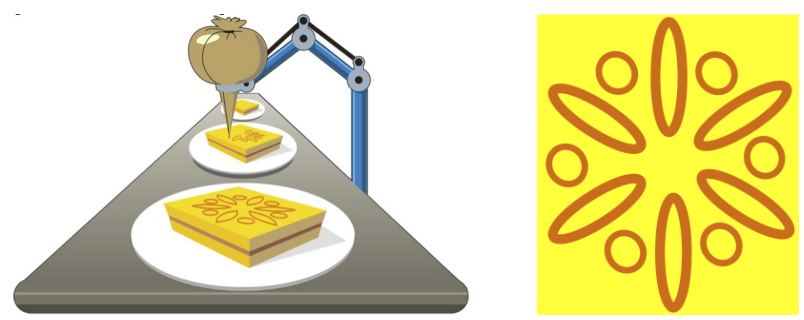

Fig. 2. Task 2015-LT-03. 


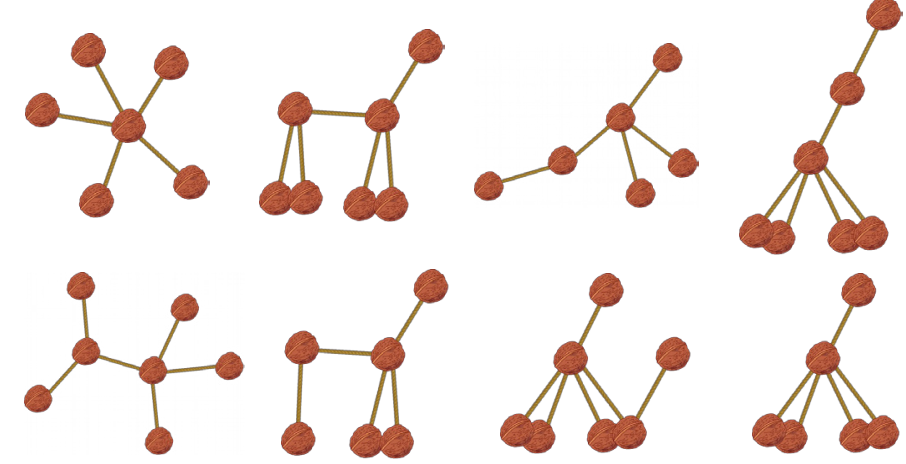

Fig. 3. Task 2015-CZ-01.

Further examples are the tasks 2015-DE-07, asking for a liquid-pouring plan using three different beakers to attain a fair share of a chemical, and 2015-HU-05, essentially a towers of Hanoi puzzle of height three. Both tasks are clearly in the ALG class. However, the former was also classified as simulation (SIM), as the question is interactive so that student can try any number of plans before validating the solution. The latter was assigned the additional problem decomposition (PD) category, since solving the puzzle requires to figure out how freeing the largest items as well as the destination of the tower as unavoidable subproblems.

\subsection{Quantitative Analysis}

The quantitative analysis aims to gain further insight into the factors that impact on task performance. Firstly, we looked at each country's performance as a whole and saw if the estimated difficulty matches the performance. We also looked at other factors that are related to task performance such as age, gender country and type of task. In particular, we reconsidered the impact of gender in performance to see if our multinational cohorts exhibit the same trends identified in previous studies. In order to accomplish these goals, we exploited standard statistics tools (average/mean, clustering and correlation) as per data shown in subsection 4.2 .

\section{Results}

\subsection{Results of Qualitative Analysis}

We now consider the results of the classification into CT categories. $77 \%$ of the tasks belong to the algorithms \& procedures (ALG) category and $42 \%$ to data representation (DR), with $25 \%$ of the tasks covering both categories (note only $6 \%$ of the dataset does not match either ALG or DR). About one fourth of the tasks, on the other hand, 
are mapped to at least one of the remaining categories reported in Table 1, including in addition digital literacy, with a slight predominance of abstraction (ABS: 8\%) and simulation (SIM: 7\%) due to the usage of interactive tasks. In other words, the Bebras challenge is mainly focused on algorithmic thinking and data representation.

A finer-grained picture of the distribution of CT categories over age groups as well as over individual challenges is depicted in the Fig. 4 and Fig. 5, which also include the concepts data analysis (DA), problem decomposition (PD) and parallelization (PAR); note there were no instances of data collection (DC) or automation (AUT). From Fig. 4

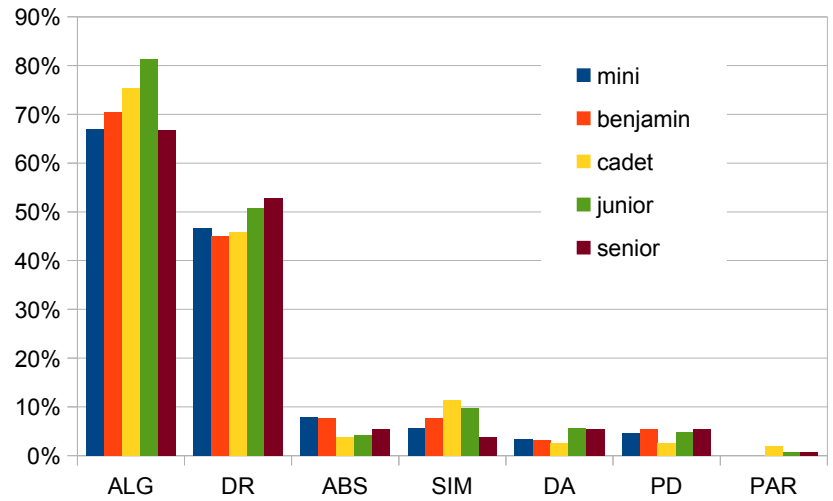

Fig. 4. Percentage of the CT categories ALG, DR, ABS, SIM, DA, PD and PAR assigned in the contests over age groups.
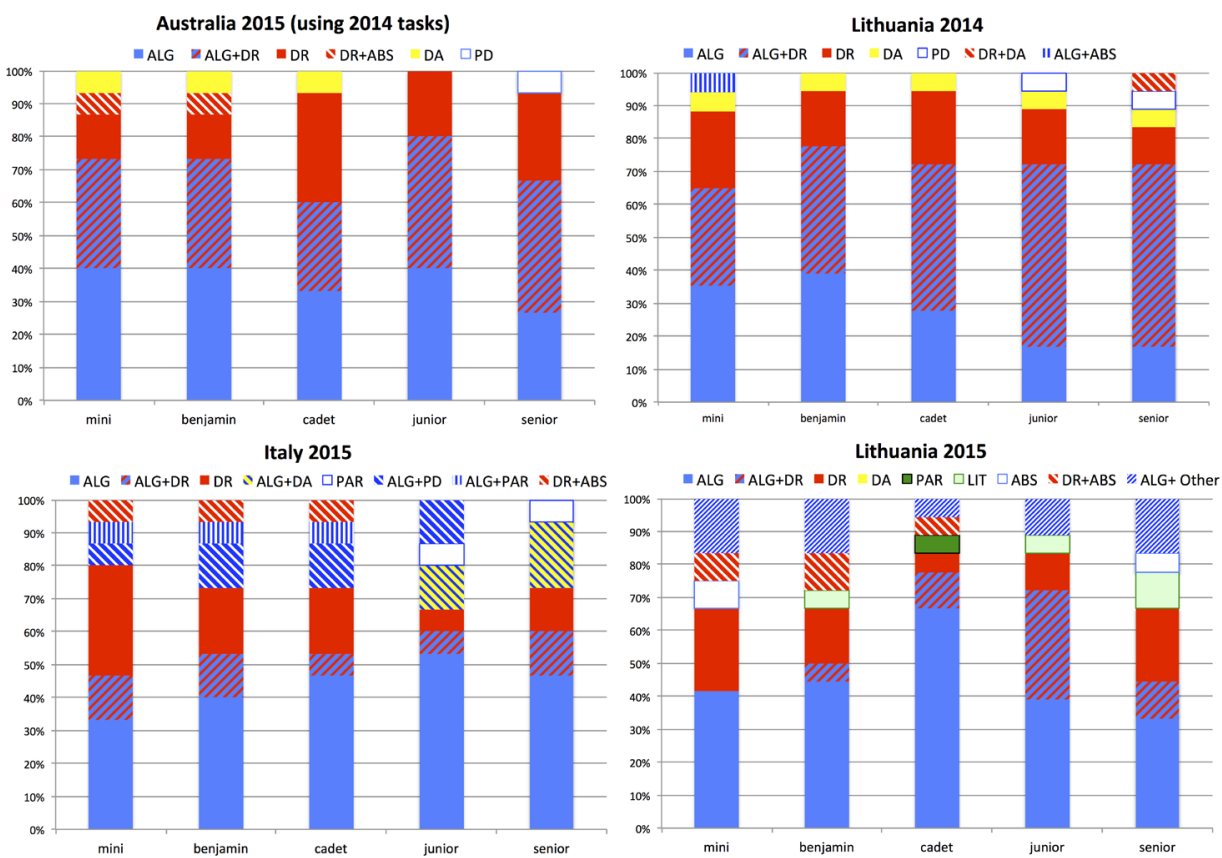

Fig. 5. Percentage of the CT categories as seen by students in 4 of the 7 country challenges. 
we can see that the percentage of tasks classified as ALG varies between $67 \%$ (Mini and Senior) and $81 \%$ (Junior), whereas the percentage relative to DR falls in the range $45 \%-53 \%$, achieving the maximum for Senior. It may also be interesting to observe that the largest percentage of tasks implying the ABS category $(8 \%)$ can be found in the Mini or Benjamin challenges, most often together with DR.

The plots in Fig. 5, on the other hand, show what is the distribution seen by students in each age group taking a contest in four of the sample countries ${ }^{9}$. Overall, ALG tasks (either alone or combined with other categories) represent at least $2 / 3$ of the tasks assigned for each age-group in any country, ranging from about $67 \%$ (Switzerland 2015) to more than $93 \%$ (Canada 2015). The incidence of different CT categories appears to depend much more on the year of the task dataset than on the country. As observed in Fig. 5, the percentage of tasks classified DR (including ALG+DR) dropped by nearly half from 2014 to 2015, replaced by more ALG tasks and with additional but limited exposure to tasks from other categories, i.e. ABS, PD and PAR.

Algorithmic tasks. Algorithmic thinking encompasses a diverse range of algorithms, and as it covers $2 / 3$ of the Bebras tasks, it may be interesting to describe in more detail the range of questions that belongs to this category. Analyzing the $474 \mathrm{ALG}$ items used in the 42 considered challenges ${ }^{10}$, we observed the following subtypes and distributions:

- $9 \%$ of the questions require to understand or produce short sequences of commands to achieve some goal; as it may be expected, the bulk of these questions $(82 \%)$ were addressed to the Mini and Benjamin age groups.

- $18 \%$ of the tasks ask students to devise less straightforward (program) strategies or plans, such as those needed to carry out a dichotomic search, to instruct a "turtle" to draw a shape, or to solve a tower-of-Hanoi puzzle.

- $12 \%$ are either about mastering or combining higher-level (e.g. procedural) behavior patterns in a process, $83 \%$ of which were addressed to the Cadet, Junior and Senior age groups.

- $26 \%$ of them require to carry out some combinatorial analysis and/or to figure out strategies to reduce complexity. Half of them have also been classified as DR: typical such instances, e.g., ask for an optimal path in a graph according to some given criteria. $81 \%$ of these tasks were again addressed to the last three age groups.

- Other kinds of task (23\% of the items overall) include understanding how data are coded, tracking the execution of a program based on some form of evolving state, carrying out accurate deductions.

\subsection{Results of Quantitative Analysis}

Task difficulty versus success rate. Each age group attempts three sets of (5 or 6) tasks, ranked in increasing difficulty as A (easy), B (medium) and C (hard). We have considered the following mapping between a priori rank and success rate: A means around 3/4

\footnotetext{
9 The others are not shown due to space limitation.

${ }^{10}$ Here the same task is counted as many times as there are contests which include it.
} 
of the class will solve the task correctly, B means $1 / 2$ of the class will solve the task, and C means only $1 / 4$ of the class will solve the task.

Fig. 6 shows the success rate versus the expected range of success for the easy, medium and hard questions for each challenge. Ideally, all results should fit inside the two trend lines plotted in the chart. Points above reflect questions easier than estimated; points below reflect harder questions. Overall, Fig. 6 seems to indicate that each contest has a reasonable mix of questions, although the rank given to several tasks does not match the observed performance. A detailed analysis of the difficulty of each contest is provided in Table 3 . Ideally, we should have $33 \%$ of questions in each actual easy/

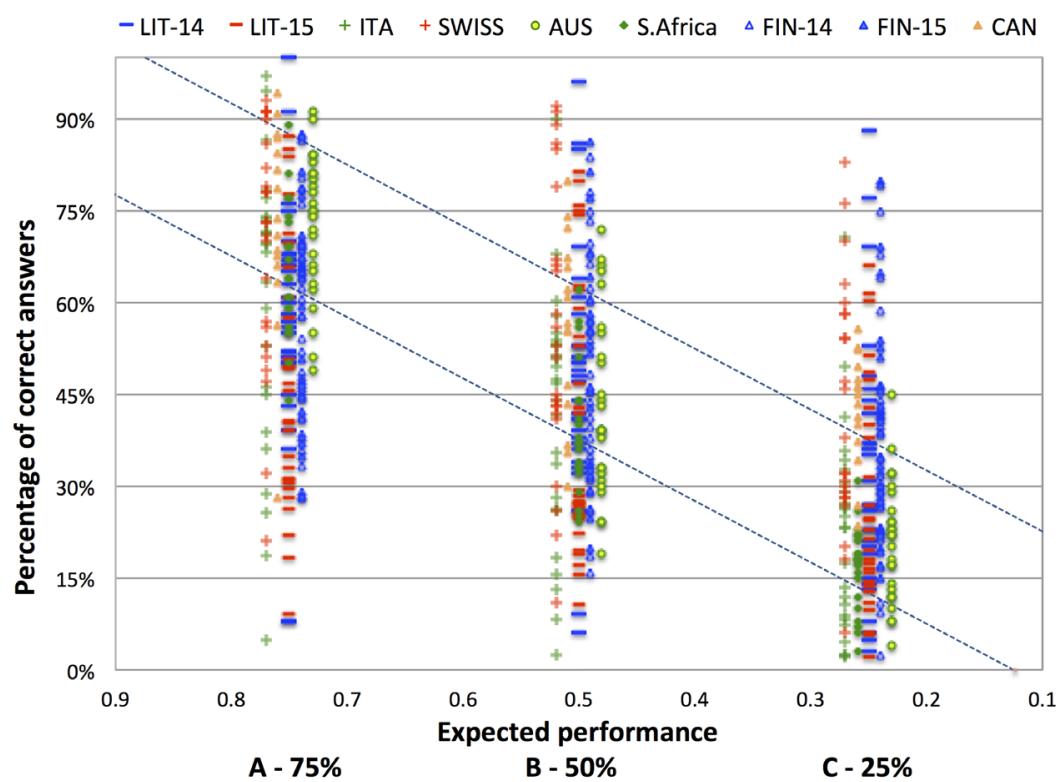

Fig. 6. Expected versus real success rate for $\mathrm{A} / \mathrm{B} / \mathrm{C}$ tasks in each dataset.

Table 3

Spread of a-posteriori easy/medium/hard tasks for each challenge (aiming for 33\% each), tasks estimated at the right level, and average success rate per country

\begin{tabular}{llllll}
\hline Set & $\begin{array}{l}\text { easy } \\
>0.65\end{array}$ & $\begin{array}{l}\text { medium } \\
0.65-0.35\end{array}$ & $\begin{array}{l}\text { hard } \\
<0.35\end{array}$ & $\begin{array}{l}\text { Rank } \\
\text { match }\end{array}$ & $\begin{array}{l}\text { Avge } \\
\text { success }\end{array}$ \\
\hline SW & $36 \%$ & $39 \%$ & $25 \%$ & $52 \%$ & $55.8 \%$ \\
ITA & $23 \%$ & $32 \%$ & $45 \%$ & $65 \%$ & $41.2 \%$ \\
FIN-14 & $23 \%$ & $49 \%$ & $28 \%$ & $45 \%$ & $47.3 \%$ \\
FIN-15 & $26 \%$ & $40 \%$ & $34 \%$ & $49 \%$ & $49.2 \%$ \\
LIT-14 & $19 \%$ & $51 \%$ & $30 \%$ & $53 \%$ & $45.1 \%$ \\
LIT-15 & $15 \%$ & $31 \%$ & $54 \%$ & $40 \%$ & $38.6 \%$ \\
CAN & $38 \%$ & $51 \%$ & $11 \%$ & $53 \%$ & $57.4 \%$ \\
AUS & $28 \%$ & $29 \%$ & $43 \%$ & $72 \%$ & $45.6 \%$ \\
SAf & $15 \%$ & $38 \%$ & $47 \%$ & $68 \%$ & $40.2 \%$ \\
\hline
\end{tabular}


medium/hard rank. The Lithuanian challenge in 2015 appears to be the hardest. Of the 84 tasks used among the five age groups, 36 were harder than estimated and 14 were easier, with only 34 tagged at the right difficulty level. On the other hand, the Australian has a more balanced distribution with only 16 harder and 5 easier tasks out of the 75 tasks used.

Success rates by country. The top histogram in Fig. 7 show the average success rates for the common tasks shared between the different challenges using the 2014 tasks. The point of this graph is not to compare countries, but to put in context the numbers given in Table 3. The bottom histogram shows the detail for the cadet 2014 contest, which has 12 common tasks for the 4 countries. As seen by that plots, when we look at the Cadet group, only a few tasks such as 2014-JP-03 (easy) or 2014-DE-05 (hard) can be considered at the same level for all four countries, while other tasks such as 2014-FR-03 or 2014-JP-06 are hard for some countries and medium or easy for others. Lithuania,

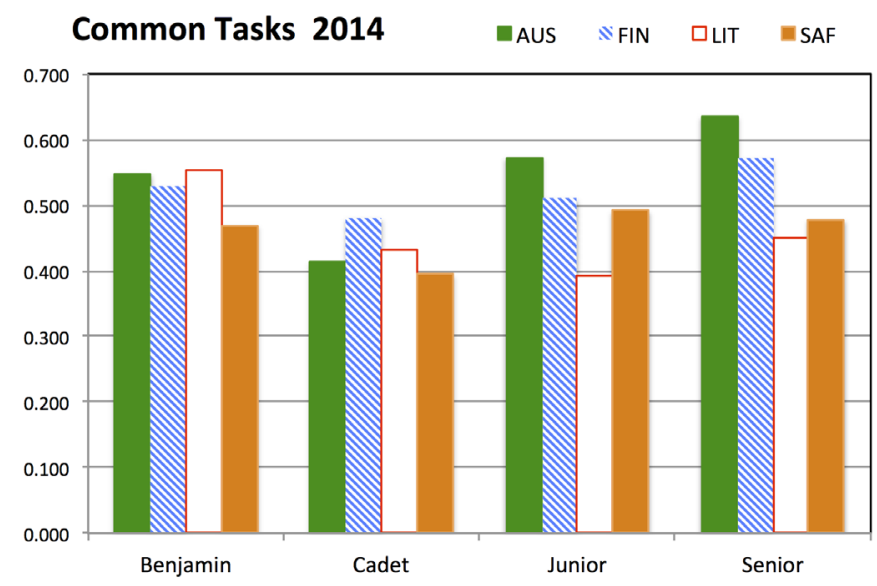

Common Tasks- Detail Cadet 2014

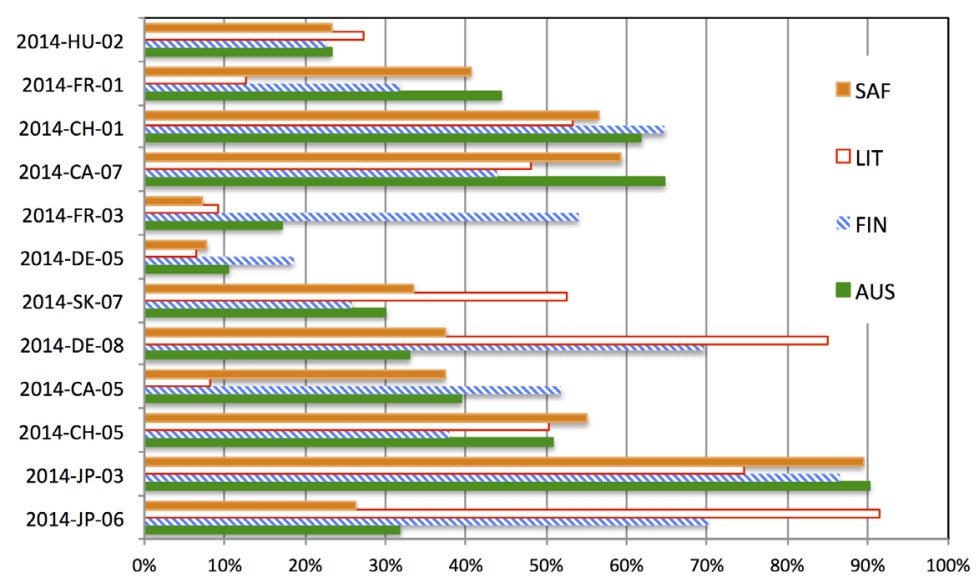

Fig. 7. Average success rate by age groups (top) and task success rate for the Cadet group (bottom) for the list of common 2014 tasks. 
for some particular reason seems to perform the best in some problems while doing poorly in others. This variability is also observed in other age groups, both in the 2014 and 2015 challenges.

Task performance by task types. The average performance by CT category for each age group varied between $40 \%$ and $50 \%$ with few exceptions. In particular, the success rate in ALG tasks ranged from 42\% (Mini and Junior) to $45 \%$ (Cadet); in DR tasks from $42 \%$ (Mini) to $50 \%$ (Cadet). Overall, DR and DR+ALG tasks turned out to be easier than purely ALG tasks. The only exception arises with the Mini age group, who showed significantly lower performance (37\%) in the problems assigned to both the ALG and DR classes, probably due to the cognitive load of mastering procedures and data at the same time. We also observed low performance for questions requiring data analysis or simulation, but the number of tasks was very small.

By considering the structure of typical ALG tasks outlined in subsection 4.1, the tasks requiring to think of a process as a combination of "behavior patterns" turned out to be the hardest ones, with a success rate around $31 \%$ in average. In particular, an extremely difficult instance was 2015-CH-09. This problem described four simple local rules to navigate a maze and asked which one of them allowed to escape a given maze. It was assigned in Italy and resulted in 2.2\% (Cadet), 2.3\% (Junior) and 4.9\% (Senior) success rate. Also the questions asking to detail a non trivial plan to achieve a goal appeared to be rather challenging ( $36 \%$ average success rate), especially for the three younger age groups. One such example is 2014-FR-03, whose description can be found in subsection 5.3. On the other hand, perhaps not surprisingly, the easiest questions are those asking for short sequences of commands to achieve a goal (56\% average success rate), that were mostly proposed to Mini and Benjamin students.

Gender participation and impact on success rate. All countries show similar patterns on gender participation, with a balanced participation at lower levels, Mini to Cadet age groups, but showing higher participation of boys both in the Junior and in the Senior level, as shown in Table $4^{11}$.

Table 4

Gender participation data per country

\begin{tabular}{|c|c|c|c|c|c|c|c|c|c|c|}
\hline \multirow[t]{2}{*}{ Set } & \multicolumn{2}{|c|}{ Mini } & \multicolumn{2}{|c|}{ Benjamin } & \multicolumn{2}{|c|}{ Cadet } & \multicolumn{2}{|c|}{ Junior } & \multicolumn{2}{|c|}{ Senior } \\
\hline & $\% \mathrm{~F}$ & $\% \mathrm{M}$ & $\% \mathrm{~F}$ & $\% \mathrm{M}$ & $\% \mathrm{~F}$ & $\% \mathrm{M}$ & $\% \mathrm{~F}$ & $\% \mathrm{M}$ & $\% \mathrm{~F}$ & $\% \mathrm{M}$ \\
\hline SW & 45 & 55 & 46 & 55 & 49 & 51 & 50 & 50 & 36 & 63 \\
\hline ITA & 45 & 55 & 44 & 56 & 44 & 56 & 23 & 77 & 21 & 79 \\
\hline FIN-14 & 52 & 47 & 45 & 55 & 48 & 52 & 34 & 66 & 10 & 90 \\
\hline FIN-15 & 51 & 49 & 51 & 49 & 46 & 53 & 41 & 59 & 30 & 70 \\
\hline LIT-14 & 43 & 56 & 46 & 54 & 44 & 56 & 42 & 58 & 30 & 70 \\
\hline LIT-15 & 45 & 55 & 47 & 53 & 44 & 56 & 43 & 57 & 31 & 69 \\
\hline CAN & - & - & - & - & 43 & 52 & 38 & 62 & - & - \\
\hline AUS & 48 & 40 & 48 & 35 & 43 & 40 & 26 & 55 & 15 & 70 \\
\hline SAf & - & - & 51 & 46 & 44 & 53 & 26 & 71 & 29 & 68 \\
\hline
\end{tabular}

${ }^{11}$ If total is less than $100 \%$, there was a "not reported" option. 
Regarding the impact of gender on success rate, the correlation of performance patterns (\% success) of girls vs. boys is high, ranging from 0.935 (SAf) to 0.98 (LIT). This means that task difficulty levels are essentially the same for both female and male students. Fig. 8 plots the success rate of female versus male students for each question (dashed line in the plot corresponds to boys being twice as successful as girls). Most data points are very close to the diagonal, witnessing similar performance regardless of gender. However, there are more outliers above than below the diagonal, indicating a minor advantage for boys in some tasks, a selection of which we will explore later. This feature is almost unnoticeable for the Mini, Benjamin and Cadet age groups (red points in the plot), becomes a little more evident for Junior (blue), and emerges clearly for Senior (green).

Interestingly, by drawing linear interpolations relative to each country and age group (not shown in the picture), we would notice that on the hardest tasks boys' performance tends to be just slightly better than that of girls - whereas the opposite happens for the easiest questions relative to the Mini-Cadet age groups. This observation appears to be consistent for all countries.

By comparing the percentages of successful tasks of girls vs. boys, there is a clear trend of worsening performance of girls relative to boys from Mini to Senior age. At

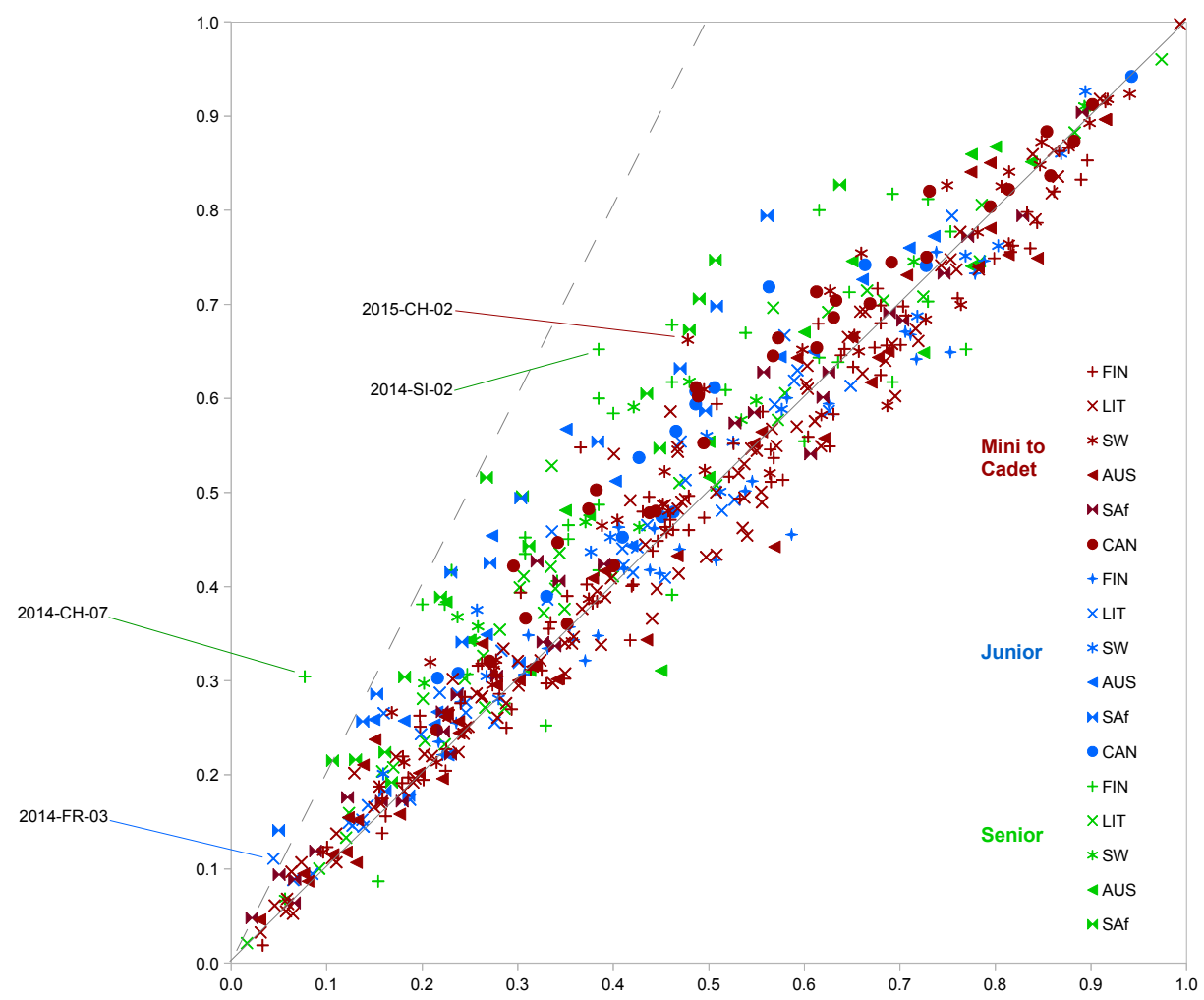

Fig. 8. Task success rate correlation of girls (horizontal axis) vs. boys, with sample outliers labeled with their relative task code. 
Senior level boys significantly outperform girls in all countries, the difference in average success rate varying from $3.0 \%$ (LIT 2015) to $15.0 \%$ (SAf). We observed gender variations by country as follows:

- The average success rate is systematically in favor of boys in Canada (from 5.5\% to $7.3 \%$ ), South Africa (from $0.2 \%$ to $15 \%$ ) and Switzerland (from $0.5 \%$ to $6.9 \%$ ), whereas in Australia (from $-2.3 \%$ to $7.7 \%$ ) the only exception are Cadet.

- The results are more balanced in Finland and Lithuania, where girls perform better than boys in several contests. Apart from Senior, the difference of the average percentages of successful tasks is usually rather small in these countries, but it should be noticed that also a deviation of about $1 \%$ can be statistically significant for a large number of participants. For example, this is the case in favor of Cadet boys for LIT 2015: based on the outcome of Welch's t-test, the difference $(1.0 \%)$ is statistically significant with $p$-value $=0.020$.

\section{Discussion}

\subsection{CT Categories}

The categorization outlined in subsection 4.1 covers unevenly the range of CT concepts in the chosen framework. At first this makes sense in that both algorithms and data representation are core concepts at introductory level. Moreover, it is not easy to figure out tasks addressing data collection or automation while meeting the strict constraints of the challenge. From a pedagogical perspective, however, we should ask what may be an "ideal coverage" of CT concepts and a balanced approach as to the other key concepts, in particular abstraction and problem decomposition. Abstraction, for instance, is usually considered more advanced and we may expect, on the one hand, that only a small percentage of tasks involve it. On the other hand, it is surprising that the majority of such tasks can be found in the challenges addressed to the youngest age groups, to whom abstraction should be especially hard. So, perhaps there is room for designing more tasks implying abstraction - or problem decomposition - at an appropriate level of difficulty.

Our previous work (Barendsen et al., 2015) categorized the task pool for the 2010 2014 and found similar dominance of ALG and DR tasks. This paper extends that analysis by looking at individual country selections for each age group and their associated performance.

\subsection{Task Difficulty}

When examining the charts in Fig. 7 we can note that, in average, the medium tasks are targeted around the $50 \%$ success for most age groups and countries. Overall, however, there are fewer easy tasks than intended. On the other hand, although the bulk of hard 
tasks have success rates in expected range, this is usually due to the presence of one or two easy tasks that lift their average for almost all age groups.

Assessing the difficulty of new tasks is a challenging process, and the estimated ranks exhibited mixed success. Our observations are consistent with previous studies pinpointing a discrepancy between intended and actual difficulty level, regardless of age group or country (Bellettini et al., 2015, van der Vegt, 2013).

Note that those appreciable performance differences between countries for some of the common tasks indicate that an estimation of task difficulty based on CT category alone will only be effective for some problems, and there are other factors that caused such variable performance. It is hard to speculate what these factors will be, as if differences are caused by students background knowledge or familiarity with the context of the examples, the variations will be more consistent within a given country.

\subsection{Gender}

The Bebras challenge is equally popular among boys and girls in the early years, while girls' participation slowly decreases in relation to that of boys starting around age 12 . This observation is likely related to the fact that "the gender gap in STEM orientations is largely a process that occurs during the high school years" (Legewie and DiPrete, 2011), but there may be other potential reasons for this change. The overall participation rate is indeed smaller for Junior and Senior compared to the younger groups. At the same time, older pupils are more likely to have developed a personal "identity" and wish to choose the activities to get involved in. In addition, the challenge may be more commonly delivered in specific STEM courses, where females are underrepresented (Justman and Méndez, 2016, Office for Civil Rights \& U.S. Department of Education, 2012).

The graph in Fig. 8 shows that, overall, the difference in performance between boys and girls was usually small, hence supporting the notion that there is nothing specifically "male" about computational thinking. On the other hand, our findings are more in line with the results of the German study discussed in (Hubwieser et al., 2016) than with the patterns observed by (Dagiene et al., 2014). In Finland and Lithuania the performance of girls vs. boys is fairly balanced, especially for the first three age groups, but overall the situations where girls outperform boys seem to be more of an exception than a rule.

When examining the plotted data, we identified a few tasks on which boys performed definitely better than girls in several countries. Those tasks required spatial skills and also relied on pupils having an intuitive understanding of binary search, which in its turn does have spatial implications to make a mental image of what is going on. Gender differences in spatial ability are well known, and have been investigated both in general (Linn and Petersen, 1985) and in the specific case of solving Bebras problems (Dagienè et al., 2015). However, even for some of the tasks requiring spatial abilities, the performance of boys were unequivocally better only at the Junior and Senior levels. Here is a selection of the tasks for which boys performed better than girls: 
The largest relative difference of performance between boys and girls is attained in 2014-CH-07 (about 75\% for FIN Senior), a task where students are asked to choose from a list of four "turtle" programs the only one which does not produce a given drawing - a composition of 5 squares of different size and color. The gender impact is less extreme but also large in other countries (40\% for SAf Senior and just below $30 \%$ for SAf Junior and AUS Senior).

In 2014-FR-03 the student should find a hidden object in a labyrinth by revealing parts of the labyrinth by clicking in it as few times as possible. The way in which clicking the labyrinth reveals it is shown as one starts clicking. The least number of clicks is the result of using a binary search strategy. (Gender differences: SAf Benjamin 55\%, SAf Cadet: 47\%, AUS Mini: 35\%, AUS Cadet: 34\%, AUS Junior: 42\%, LIT Cadet: 31\%, LIT Junior: 60\%).

In 2015-CH-02 the student should navigate to a given room number by going either to the left or to the right from the current room number. The goal is to find the correct room with as few movements as possible. The best result is achieved by using binary search. (Gender differences: FIN Benjamin: 23\%, FIN Cadet: 33\%, LIT Benjamin: 26\%, LIT Cadet: 22\%, SW Benjamin: 27\%).

In 2014-SI-02 the student is given a two-dimensional simple labyrinth in which a robot should find a given object starting from a given position. The student is to find out which of four lists of commands written in alien language can take the robot to the element. (Gender differences: FIN Senior: $41 \%$, SAf: $25-31 \%$ in the two oldest age groups).

\section{Conclusions}

This paper has thoroughly analyzed the CT content of Bebras tasks assigned in the 2014-2015 challenges as well as the related performance of a large multinational cohort of students. While trying to answer the third research question introduced in Section 1, our study has validated some previous observations based on either smaller or less heterogeneous samples:

(1) Overall, students from different school systems exhibit comparable CT skills.

(2) A declining performance trend of girls vs. boys can be recognized from primary to high school level, with boys outperforming girls in all countries at Senior age.

(3) Gender differences relative to all age groups were observed for some tasks related to spatial skills.

How to reliably estimate the difficulty of new tasks is still an open issue, for which we need to better understand and characterize the range of algorithmic strategies used in the challenge, and the factors that caused large task performance variations among countries. The CT conceptual content, in particular, does not seem to play a major role in assessing how difficult a task is (second research question). However, the fact that young students around the world managed to solve many Bebras tasks prior to having received any instruction on CT reflects their readiness to learn core CT concepts using everyday examples. 
Our data indicate that the challenge mainly addresses the ALG and DR categories (first research question). This is to be expected on the lower age groups, but we should provide Junior and Senior students with a variety of tasks that significantly covers other CT concepts/skills. Our suggestion to the Bebras community may be to try to take a broader perspective on CT in the future. Besides, creating an archive of Bebras tasks which can be searched by CT subcategory may improve the use of the challenge as a tool to teach CT concepts in the classroom.

\section{References}

Barendsen, E., Grgurina, N., Tolboom, J. (October 13-15 2016). A new informatics curriculum for secondary education in the Netherlands. In: Brodnik, A., Tort, F. (Eds.), Proc. of the 9th International Conference on Informatics in Schools: Situation, Evolution, and Perspectives, ISSEP 2016. Springer International Publishing, Cham, pp. 105-117.

Barendsen, E., Mannila, L., Demo, B., Grgurina, N., Izu, C., Mirolo, C., Sentance, S., Settle, A., Stupurienė, G. (2015). Concepts in K-9 computer science education. In: Proc. of ITiCSE on Working Group Reports. ITICSE-WGR'15. ACM, pp. 85-116.

Barendsen, E., Steenvoorden, T. (October 13-15 2016). Analyzing conceptual content of international informatics curricula for secondary education. In: Brodnik, A., Tort, F. (Eds.), Proc. of the 9th International Conference on Informatics in Schools: Situation, Evolution, and Perspectives, ISSEP 2016. Springer Int. Publishing, Cham, pp. 14-27.

Bell, T., Curzon, P., Cutts, Q., Dagienė, V., Haberman, B. (2011). Overcoming obstacles to CS education by using non-programming outreach programmes. In: Proc. of the 5th ISSEP Conf. Springer-Verlag, pp. 71-81.

Bellettini, C., Lonati, V., Malchiodi, D., Monga, M., Morpurgo, A., Torelli, M. (2015). How challenging are Bebras tasks? An IRT analysis based on the performance of Italian students. In: Proc. of ITiCSE '15. ACM, pp. 27-32.

Bezáková, D., Winczer, M. (2011). Teaching theoretical informatics to secondary school informatics teachers. In: Proc. of the 5th ISSEP Conf. Springer Berlin Heidelberg, pp. 117-128.

Cohen, L., Manion, L., Morrison, K. (2013). Research Methods in Education. London, New York: Routledge.

Dagienè, V. (2006). Information technology contests - introduction to computer science in a attractive way. Informatics in Education, 5(1), 37-46.

Dagienè, V., Mannila, L., Poranen, T., Rolandsson, L., Söderhjelm, P. (2014). Students' performance on programming-related tasks in an informatics contest in Finland, Sweden and Lithuania. In: Proc. of ITiCSE'14. ACM, pp. 153-158.

Dagienè, V., Mannila, L., Poranen, T., Rolandsson, L., Stupurienė, G. (2014). Reasoning on children's cognitive skills in an informatics contest: Findings and discoveries from Finland, Lithuania, and Sweden. In: Proc. of the 7th ISSEP Conf. Springer Int. Publishing, pp. 66-77.

Dagienè, V., Pèlikis, L., Stupurienè, G. (2015). Introducing computational thinking through a contest on informatics: Problem-solving and gender issues. Informacijos Mokslai, 73, 43-51.

Dagienè, V., Sentance, S. (October 13-15 2016). It's Computational thinking! Bebras tasks in the curriculum. In: Brodnik, A., Tort, F. (Eds.), Proc. of the 9th International Conference on Informatics in Schools: Situation, Evolution, and Perspectives, ISSEP 2016. Springer Int. Publishing, Cham, pp. 28-39.

Dagienė, V., Stupurienė, G. (2016). Bebras - a sustainable community building model for the concept based learning of informatics and computational thinking. Informatics in Education, 15(1), 25-44.

Duncan, C., Bell, T. (2015). A pilot computer science and programming course for primary school students. In: Proc. of WiPSCE '15. ACM, pp. 39-48.

Gavora, P. (2015). The state-of-the-art of content analysis. Education Sciences, 1, 6-18.

Gujberova, M., Kalas, I. (2013). Designing productive gradations of tasks in primary programming education. In: Proc. of the 8th WiPSCE Conf. ACM, pp. 108-117. 
Haberman, B., Averbuch, H., Cohen, A., Dagienè, V. (2011). Work in progress - Initiating the Beaver contest on computer science and computer fluency in Israel. In: Proc. of the Frontiers in Education Conference. pp. T1D:1-2.

Heintz, F., Mannila, L., Färnqvist, T. (October 2016). A review of models for introducing computational thinking, computer science and computing in K-12 education. In: Proceedings of the 46th Annual Frontiers in Education, FIE 2016. pp. 1-9.

Hubwieser, P., Armoni, M., Giannakos, M.N., Mittermeir, R.T. (2014). Perspectives and Visions of Computer Science Education in Primary and Secondary (K-12) Schools. Transactions on Computing Education, 14(2), 7:1-7:9.

Hubwieser, P., Hubwieser, E., Graswald, D. (October 13-15 2016). How to attract the girls: Gender-specific performance and motivation in the Bebras challenge. In: Brodnik, A., Tort, F. (Eds.), Proc. of the 9th International Conference on Informatics in Schools: Situation, Evolution, and Perspectives, ISSEP 2016. Springer Int. Publishing, pp. 40-52.

Hubwieser, P., Mühling, A. (2014). Playing PISA with Bebras. In: Proc. of the 9th WiPSCE Conf. ACM, pp. $128-129$.

Hubwieser, P., Mühling, A. (2015). Investigating the psychometric structure of Bebras contest: Towards mesuring computational thinking skills. In: Proc. of the Int. Conf. on Learning and Teaching in Computing and Engineering. pp. 62-69.

Justman, M., Méndez, S.J. (March 2016). Gendered selection of STEM subjects for matriculation. Melbourne Institute of Applied Economic and Social Research - Working Paper No. 10/16.

Kalelioglu, F., Glbahar, Y., Kukul, V. (2016). A framework for computational thinking based on a systematic research review. Baltic Journal of Modern Computing, 4(3), 583-596.

Legewie, J., DiPrete, T.A. (July 2011). High School Environments, STEM Orientations, and the Gender Gap in Science and Engineering Degrees. Tech. rep., Columbia University Academic Commons.

Linn, M.C., Petersen, A.C. (1985). Emergence and characterization of sex differences in spatial ability: A meta-analysis. Child Development, 56(6), 1479-1498.

Mannila, L., Dagienė, V., Demo, B., Grgurina, N., Mirolo, C., Rolandsson, L., Settle, A. (2014). Computational thinking in K-9 education. In: Proc. of ITiCSE on Working Group Reports. ITiCSE-WGR'14. ACM, pp. 1-29.

McCartney, R., Tenenberg, Eds., J. (June 2014). Trans. on Computing Education - Special Issue on Computing Education in K-12 Schools, 14(2).

Office for Civil Rights \& U.S. Department of Education (June 2012). Gender Equity in Education - A Data Snapshot.

Papert, S. (1980). Mindstorms: Children, Computers, and Powerful Ideas. Basic Books, Inc., New York, NY, USA.

Sentance, S., Dorling, M., McNicol, A. (February 26-March 2 2013). Computer science in secondary schools in the UK: Ways to empower teachers. In: Diethelm, I., Mittermeir, R.T. (Eds.), Proc. of the 6th Int. Conference on Informatics in Schools: Situation, Evolution, and Perspectives, ISSEP 2013. Springer, Berlin, Heidelberg, pp. 15-30.

Tort, F., Drot-Delange, B. (2013). Informatics in the French secondary curricula: Recent moves and perspectives. In: Proc. of the 6th ISSEP Conf. Springer, Berlin, Heidelberg, pp. 31-42.

van der Vegt, W. (2013). Predicting the difficulty level of a Bebras task. Olympiads in Informatics, 7, 132-139.

Wing, J.M. (2006). Computational thinking. Communications of the ACM, 49(3), 33-35.

Yagunova, E., Podznyakov, S., Ryzhova, N., Razumovskaia, E., Korovkin, N. (2015). Tasks classification and age differences in task perception: Case study of international on-line competition "Beaver". In: Proc. of the 8th ISSEP Conf. Univ. of Ljubljana, pp. 33-43. 
C. Izu is a lecturer in Computer Science at the University of Adelaide. She leads the outreach program CS4HS which helps middle and high school teachers introduce Computer Science and computational thinking to their classrooms. She has been a curriculum writer for the new CT subject (Year 11-12) in her state, to be rolled out in 2018. Besides her regular research in interconnection networks, Cruz is currently exploring the learning issues of novice programmers.

C. Mirolo is researcher in Computer Science at the University of Udine (Italy), Dept. of Mathematics, Computer Science and Physics, where he currently teaches introductory programming and computational geometry. He is also responsible for the education/ training programmes offered to prospective high school teachers of CS. His research interests include students' learning of programming and the role of computational thinking in general education.

A. Settle is an associate professor in the School of Computing in the College of Computing and Digital Media at DePaul University in Chicago, Illinois, USA. She teaches computer science, focusing primarily on introductory programming courses. Her research interests include computational thinking, game development and design, gender and computing, online learning, programming pedagogy, and student recruitment and retention.

L. Mannila is a researcher in computer science education at university in Linköping, Sweden. Her research interests include questions related to computational thinking, digital competence and programming at K-9 level, both from a student and a teacher perspective. She leads several projects related to programming didactics and developing suitable teaching materials for introducing computational thinking at lower levels of education. Mannila is also involved in organizing the Bebras contest in Finland and Sweden.

G. Stupurienè is a doctoral student at Vilnius University Institute of Mathematics and Informatics at the Department of Informatics Methodology. She has been working with Bebras challenge since 2010. As a Master student she worked on Conceptualisation of Informatics Fundamentals through Bebras Tasks of earlier years. Her main research focus is developing informatics concepts based educational model for schools. 